\title{
Cancer testis antigen expression in testicular germ cell tumors and in intratubular germ cell neoplasia
}

Modern Pathology (2015) 28, 742-744; doi:10.1038/modpathol.2014.144

To the Editor: I read with great interest the article by Bode et $a l^{1}$ 'Cancer testis antigen expression in testicular germ cell tumorigenesis'. As one of the initial researchers that identified this category of tumor antigens and coined the term CT antigen, ${ }^{2}$ we have also examined the expression of the CT antigens in germ cell tumors by immunohistochemical analysis, including classic seminoma, spermatocytic seminoma and non-seminomatous germ cell tumors, and these data have been published. ${ }^{3,4}$

Comparing the data of Bode et al to ours, the following are the shared conclusions: (1) spermatocytic seminomas show consistent and strong expression of almost all CT antigens, reflecting their origin from the adult spermatogonia or primary spermatocytes, as CT antigens are constitutively expressed in these adult premeiotic testicular germ cells as differentiation antigens, (2) classic seminomas express different CT antigens at variable but lower frequencies than in spermatocytic seminomas; Bode et al observed $3-40 \%$ expression rate among five CT antigens (MAGEA3, MAGEA4, GAGE1, CT7 (MAGEC1) and NY-ESO-1 (CTAG1B)), whereas we found 4-> 80\% among the seven antigens (MAGEA, NY-ESO-1, GAGE, CT7, CT10, CT45 and SAGE1) analyzed, and (3) non-seminomatous germ cell tumors expressed CT antigens much less frequently, in fact not detected at all in this study of Bode et al.

Two significant differences, however, were noted when we compared our data with those of Bode et al, specifically in regard to the expression of CT antigens in non-seminomatous germ cell tumors and in intratubular germ cell neoplasia (IGCNU).

In non-seminomatous germ cell tumors, the authors found no expression of any of the five CT antigens in the 204 specimens included in their tissue microarray, whereas we found examples of positive expression of almost all CT antigens in embryonal carcinoma and yolk sac tumor and less frequently in the few cases of teratoma and choriocarcinomas that we had. ${ }^{3}$ Although the antibodies that they used to analyze MAGEA expression were different from ours, the same commercial antibodies were used to analyze CT7 (MAGEC1), NY-ESO-1 (CTAG1B) and GAGE expression, and technical differences in the immunohistochemical assays would be unlikely to account for the observed discrepancy. Instead, we believe that this discrepancy was mostly, if not entirely, because the expression of CT antigens in these tumors is extremely heterogeneous within the tumor and would be missed by exclusively analyzing tissue microarrays (TMA). Many cases in our series showed positive staining in $<10 \%$ of the tumor cells, often present in very small clusters (Figure 1a). By using tissue microarray instead of whole sections like we did, cases with such focal CT expression could easily be missed, leading the authors to incorrectly conclude that this group of tumors is negative for the five CT antigens examined. Gjerstorff et $a l^{5}$ using whole sections, have indeed found MAGEA and GAGE expression in teratomas, supporting our finding that many CT antigens are expressed in non-seminomatous germ cell tumors, but at lower frequencies than in seminoma and often patchy in distribution.

The second discrepancy relates to whether CT antigens are expressed in IGCNU. The authors examined 20 IGCNUs and noted that all were negative for the five CT antigens analyzed. The literature on this topic has been controversial and conflicting results have been reported. Similar to this article, Stoop et $a l^{6}$ also reported that all IGCNU cells are negative for (another CT antigen) SSX, including three cases in which the invasive components were SSX positive. On the other hand, other groups have reported MAGEA expression in 13 of $15,{ }^{7} \mathrm{NY}-\mathrm{ESO}-1$ (CTAG1B) in 7 of $15,{ }^{8} \mathrm{CT} 45$ in almost all ${ }^{9}$ IGCNUs of classic seminomas, and Bode et $a l^{1}$ also mentioned in the discussion that they detected CT10/MAGEC2 expression in all IGCNU cases. To fully address this discrepancy in the literature, we have indeed used dual-color immunostaining of OCT4 (for all IGCNU cells) and MAGEA (or CT7, for any CT-positive cells) in our recently published study. ${ }^{3}$ This dual-color staining allowed us to reliably identify any IGCNU cells that are positive for CT antigens (MAGEA or CT7). Using this strategy, we found the vast majority of IGCNU cells to be CT antigen negative. However, occasional MAGEA- or CT7-positive IGCNU cells were found (in 4/18 cases examined), always co-existing with CT-negative IGCNU cells in the same tubules (Figure 1b). We concluded that CT antigens, at least MAGEA and CT7, are infrequently expressed in IGCNU and represent only a small subset of this in situ tumor cell population. The reason for previously reported discrepant results are not entirely clear, but the false-positive results might represent misinterpretation of the constitutively CT-positive residual spermatogonia in atrophic seminiferous tubules as IGCNUs, as we have found it difficult to reliably distinguish these residual spermatogonia from IGCNU cells based on histomorphology alone. Our dual-color immunostain in fact showed frequent co-existence of these two populations in the same tubules (Figure 1b). On the other hand, two 

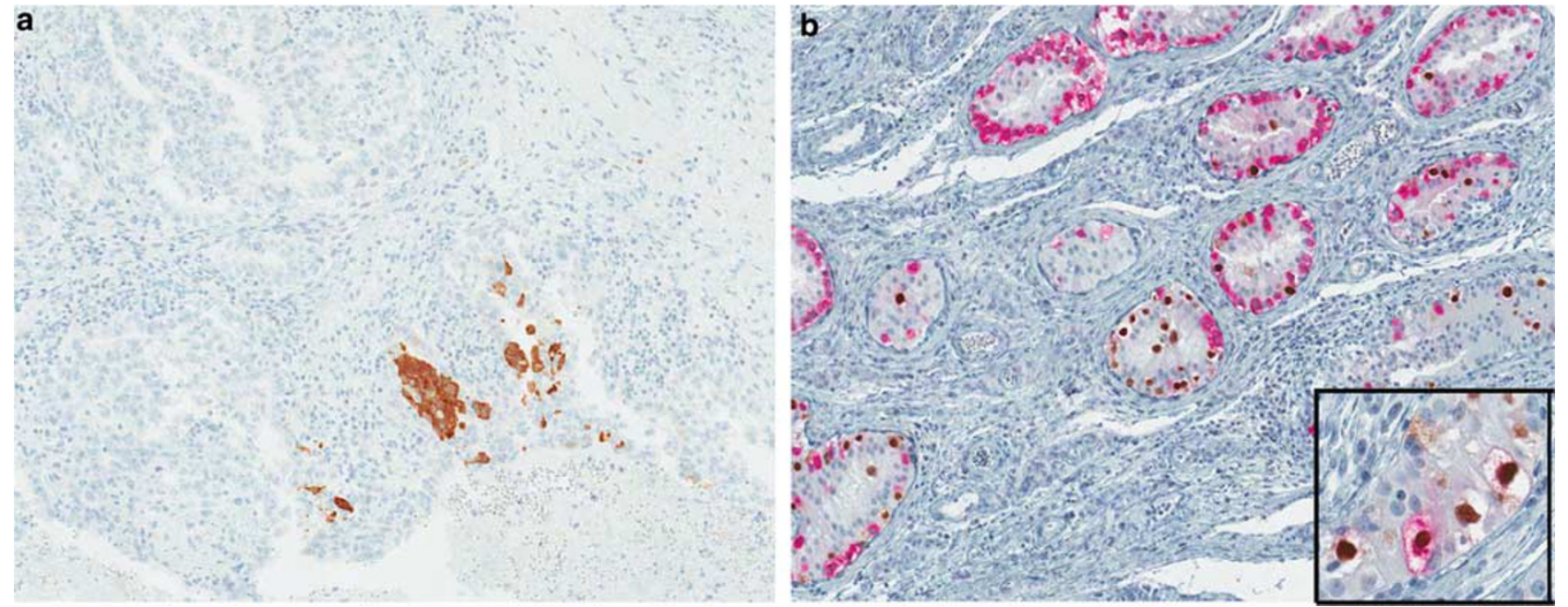

Figure 1 Expression of cancer testis (CT) antigens in non-seminomatous germ cell tumor and in intratubular germ cell neoplasia (IGCNU). (a) Expression of CT antigen, eg, MAGEA (monoclonal antibody 6C1) in this embryonal carcinoma is often very focally and in patchy distribution. (b) Dual immunostaining for OCT4 (brown nuclear staining) and CT7 (pink cytoplasmic staining) in a case of classic seminoma. Residual CT7-positive spermatogonia were seen in many atrophic tubules, some of them also contained OCT4-positive IGCNU cells. The majority of the OCT4-positive cells were CT7-negative, but occasionally CT7-positive, OCT4-positive cells can be identified (inset).

reasons might explain the false-negative results, one being limited sampling-particularly if microarray was used, and the other being attributing the positively stained but morphologically ambiguous cells as nonneoplastic germ cells rather than neoplastic IGCNU cells.

Bode et $a 1^{1}$ stated in the discussion that 'the early loss of CT antigen in intratubular germ cell neoplasia unclassified might be associated with malignant transformation and tumor progression in germ cell transformation'. On the basis of our data of CT expression in fetal testis and germ cell tumors, however, we do not believe that CT antigens are 'lost' in the IGCNU cells. Instead, we believe that primodial germ cells undergo a transition from OCT4-positive, CT-negative pluripotent cells to OCT4-negtaive, CT-positive germ cells during fetal gonad development; ${ }^{4}$ IGCNU cells are derived from cells in the earlier OCT4-positive, CT-negative stage and hence are CT negative in their undifferentiated state. These CT-negative IGCNU cells have the potential to differentiate along the germ cell lineage into classic seminoma or somatic lineages into non-seminomatous germ cell tumors, such as teratoma, yolk sac tumors etc. It is the germ cell differentiation in classic seminoma that would enable the IGCNU cells to acquire CT expression as a germ cell-specific differentiation marker, and this explains the high frequency of CT antigen expression in classic seminoma. This transition from CT-negative to CT-positive status is most often observed in invasive tumors, but can also occur during the IGCNU stage, thus the occasional observation of CT-positive IGCNU cells in our study. On the other hand, when the CT-negative IGCNU cells become non-seminomatous germ cell tumors, the vast majority of the consequent tumors would maintain their CT-negative precursor phenotype. However, these tumors may subsequently acquire CT antigen expression due to gene dysregulation in a process similar to the CT gene activation in any other somatic tumors, eg, melanoma, carcinoma, sarcoma etc. This would explain the low frequency and high intratumoral heterogeneity of CT expression in the non-seminomatous germ cell tumors, as both are known characteristics of CT antigen expression in somatic cancer.

\section{Disclosure/conflict of interest}

The authors declare no conflict of interest.

Yao-Tseng Chen ${ }^{1}$ Department of Pathology and Laboratory Medicine, Weill Cornell Medical College, New York, NY, USA E-mail: ytchen@med.cornell.edu

\section{References}

11 Bode PK, Thielken A, Brandt S, et al. Cancer testis antigen expression in testicular germ cell tumorigenesis. Mod Pathol 2014;27:899-905.

12 Chen YT, Scanlan MJ, Sahin U, et al. A testicular antigen aberrantly expressed in human cancers detected by autologous antibody screening. Proc Natl Acad Sci USA 1997;94:1914-1918.

13 Chen YT, Cao D, Chiu R, et al. Chromosome X-encoded cancer/testis antigens are less frequently expressed in non-seminomatous germ cell tumors than in seminomas. Cancer Immun 2013;13:10. 
14 Chen YT, Chiu R, Lee P, et al. Chromosome X-encoded cancer/testis antigens show distinctive expression patterns in developing gonads and in testicular seminoma. Hum Reprod 2011;26:3232-3243.

15 Gjerstorff MF, Harkness L, Kassem M, et al. Distinct GAGE and MAGE-A expression during early human development indicate specific roles in lineage differentiation. Hum Reprod 2008;23:2194-2201.

16 Stoop H, van Gurp R, de Krijger R, et al. Reactivity of germ cell maturation stage-specific markers in spermatocytic seminoma: diagnostic and etiological implications. Lab Invest 2001;81:919-928.
17 Aubry F, Satie AP, Rioux-Leclercq N, et al. MAGE-A4, a germ cell specific marker, is expressed differentially in testicular tumors. Cancer 2001;92:2778-2785.

18 Satie AP, Rajpert-De Meyts E, Spagnoli GC, et al. The cancer-testis gene, NY-ESO-1, is expressed in normal fetal and adult testes and in spermatocytic seminomas and testicular carcinoma in situ. Lab Invest 2002;82:775-780.

19 Rudolph P, Kellner U, Schmidt D, et al. Ki-A10, a germ cell nuclear antigen retained in a subset of germ cell-derived tumors. Am J Pathol 1999;154: 795-803. 